\title{
Low dielectric and low surface free energy flexible linear aliphatic alkoxy core bridged bisphenol cyanate ester based POSS nanocomposites
}

\author{
S. Devaraju ${ }^{1,2}$, P. Prabunathan ${ }^{1}$, M. Selvi ${ }^{1}$ and M. Alagar ${ }^{1 *}$ \\ 1 Polymer Composites Lab, Department of Chemical Engineering, Alagappa College of Technology, Anna University, Chennai, India \\ ${ }^{2}$ Next MEMS lab, School of Mechanical Engineering, Pusan National University, Busan, South Korea
}

Edited by:

Giuseppe Mensitieri, University of

Naples Federico II, Italy

Reviewed by:

Giuseppe Mensitieri, University of

Naples Federico II, Italy

Marino Lavorgna, Italy's National

Research Council, Italy

\section{${ }^{*}$ Correspondence:}

M. Alagar, Polymer Composites Lab, Department of Chemical

Engineering, Alagappa College of

Technology, Anna University, 223,

Chennai 600 025, India

e-mail:mkalagar@yahoo.com
The aim of the present work is to develop a new type of flexible linear aliphatic alkoxy core bridged bisphenol cyanate ester (AECE) based POSS nanocomposites for low $k$ applications. The POSS-AECE nanocomposites were developed by incorporating varying weight percentages $(0,5$, and $10 \mathrm{wt} \%$ ) of octakis (dimethylsiloxypropylglycidylether) silsesquioxane (OG-POSS) into cyanate esters. Data from thermal and dielectric studies imply that the POSS reinforced nanocomposite exhibits higher thermal stability and low dielectric value of $k=2.4$ (10 wt $\%$ POSS-AECE 4 ) compared than those of neat AECE. From the contact angle measurement, it is inferred that, the increase in the percentage incorporation of POSS in to AECE, the values of water contact angle was enhanced. Further, the value of surface free energy was lower when compared to that of neat AECE. The molecular level dispersion of POSS into AECE was ascertained from SEM and TEM analyses.

Keywords: organic-inorganic hybrid, cyanate ester, POSS, thermal properties, low-k, low surface free energy

\section{INTRODUCTION}

High-performance dielectric materials have attracted much attention in worldwide, owing to their widespread applications in many rapidly developing fields such as electric power, microelectrics, informatics, etc. (Phang et al., 2009; Jang et al., 2010; Zhuo et al., 2012). The hybridization of organic and inorganic components is the most effective method to develop high performance dielectrics, since hybridization merges the advantages of both organic and inorganic materials, and thus, has the biggest benefit to get the desired integrated performance. To date, many inorganic-organic hybrids have been investigated, and the results show that the properties of these inorganic-organic hybrids are greatly dependent on the nature of the inorganic and organic segments, and their interfacial adhesion (Laine, 2005; Liu et al., 2005; Zhang et al., 2005; Lee et al., 2006; Dang et al., 2008; Sanchez et al., 2011a,b).

The hydrophobic polymers have been a topic of considerable interest owing to their attractive properties such as low surface energy, heat resistance, chemical inertness, and low dielectric constant. Hydrophobic coatings may be used in various industrial applications including anti-wetting, anti-snow adherence, anti-rusting, reduced friction resistance etc. Low surface energy polymeric materials with good film-forming characteristics have attracted great interest because of their practical applications (Jeyaprakash et al., 2004; Yoshida et al., 2004; Qu et al., 2008). Consequently great attention has been placed on precise strategies modifying these solid surfaces. Most of the low surface energy polymeric materials that have been developed were based on flurorine- or silicon-containing polymers (Liang et al., 2011; Qu and Xin, 2011; Raza et al., 2012). Polyhedral oligomeric silsesquioxanes (POSS) have recently attracted considerable attention. The hybrid structure has significant contribution to dielectric, heat-resistant and radiation-resistant behavior of coatings. The POSS based hybrid coatings after radiation treatment forms the self-passive silica layer, which prevents further deterioration of the surface. An introduction of POSS in to polymers brings about significant improvements in their properties such as increased thermal stability, increased hardness and oxidative resistance, increased $\mathrm{Tg}$, mechanical properties and decreased flammability and viscosity during processing, increase in hydrophobicity and wear resistance as well as decrease in friction on the surfaces of the POSS-based nanocomposites (Gonzalez et al., 2000; Devaux et al., 2002; Xu et al., 2002; Leu et al., 2003; Wu et al., 2007; Tang and Lewin, 2009; Cordes et al., 2010; Nagendiran et al., 2010; Devaraju et al., 2011, 2012; Kuo and Chang, 2011; Venkatesan et al., 2011, 2012; Chandramohan et al., 2012; Zhang and Müller, 2013).

Cyanate ester (CE) resins have outstanding integrated properties, such as excellent dielectric value, thermal resistance as well as good processing characteristics etc., and hence CE resins have been regarded as potential candidates with the greatest competition to fabricate advanced functional, structural composite materials for many cutting edge fields including microelectronics, aerospace, and transportation (Fang and Shimp, 1995; Zhuo et al., 2011). CE resins are superior to conventional epoxy, polyimide, and BMI resins. For example, the moisture absorption rate of cyanate esters is lower than that of epoxy, polyimide, and BMI resins. Cyanate esters undergo thermal or catalytic cyclotrimerization to form triazine rings during curing. However, the major drawback of CE resin is its inherent brittleness, which often 
restricts its structural applications. In addition, CE needs to be cured at very high temperatures and the resins cured at high temperature tend to have higher stress concentration and internal defects, and thereby declining the integrated properties of the resultant materials in addition to high energy consumption.

In the present work, an attempt has been made to develop a series of flexible linear aliphatic alkoxy core bridged bisphenol cyanate esters based OG-POSS nanocomposites. The newly synthesized linear aliphatic alkoxy core bridged bisphenol CE monomers exhibit lower curing temperature than that of conventional CE (BADy). The OG-POSS was introduced into cyanate esters in varying weight percentages ( 5 and $10 \mathrm{wt} \%$ ), and their thermal, dielectric and morphological properties were studied.

\section{MATERIALS}

Cyanogen bromide, dibromoalkane (1,2-dibromoethane, 1,4dibromobutane, 1,6-dibromohexane, and 1,8-dibromooctane) were purchased from Spectrochem, India. 3-chloroperoxybenzoic acid (MCPBA) was purchased from Sigma Aldrich, India. 4hydroxy benzaldehyde, triethylamine, sodium carbonate, sodium sulfate, sodium hydroxide, and other solvents were obtained from SRL India and were used without further purification. OG-POSS was synthesized as per the procedure reported (Chandramohan et al., 2012; Venkatesan et al., 2012).

\section{SYNTHESIS OF 4, 4' -[ALKANE-1,2-DIYLBIS(OXY)] DIBENZALDEHYDE (AEDA)}

A two-necked round-bottomed flask, equipped with a magnetic stirrer bar, thermometer, and condenser was charged with 4-hydroxybenzaldehyde $(0.0819 \mathrm{~mol})$, dibromoalkane $(0.0409 \mathrm{~mol}), \mathrm{Na}_{2} \mathrm{CO}_{3}(0.1638 \mathrm{~mol})$, and $\mathrm{DMF}(50 \mathrm{ml})$. The mixture was stirred and heated to reflux $\left(140^{\circ} \mathrm{C}, 6 \mathrm{~h}\right)$. The reaction mixture was cooled to room temperature and quenched with distilled water and then filtered and washed with water to obtain a crude product. An off-white solid was obtained by recrystallization in ethanol, and the product was collected by filtration and dried at $50^{\circ} \mathrm{C}$ under vacuum in a hot air oven for 6 h (Scheme 1).

${ }^{1} \mathrm{HNMR}:(300 \mathrm{MHz}, \mathrm{CDCl} 3) \delta(\mathrm{ppm})$ and ${ }^{13} \mathrm{CNMR}: \delta(\mathrm{ppm})$ for

AEDA $_{1}$ : 9.9 (s, 2 H, Ar-CHO-), 7.8 (d, 4 H, ArH), 7.0 (d, 4 H, $\mathrm{ArH}$ ), 4.5 (t, 4 H, Ar-O-CH${ }_{2}$ ) and 190, 164, 132, 129, 115 (aromatic carbon), 68(aliphatic carbon).

AEDA $_{2}$ : 9.8 (s, $\left.2 \mathrm{H}, \mathrm{Ar}-\mathrm{CHO}-\right), 7.8$ (d, 4 H, ArH), 6.9 (d, 4H, $\mathrm{ArH}), 4.1\left(\mathrm{t}, 4 \mathrm{H}, \mathrm{Ar}-\mathrm{O}-\mathrm{CH}_{2}\right), 2.0\left(\mathrm{~m}, 4 \mathrm{H}, \mathrm{CH}_{2}-\mathrm{CH}_{2}\right)$ and 191, 164, 132, 130, 114 (aromatic carbon), 67, 26 (aliphatic carbon).

AEDA $_{3}$ : 9.9 (s, 2H, Ar-CHO-), 7.8 (d, 4H, ArH), 7.0 (d, 4H, ArH), 4.1 (t, 4H, Ar-O-CH $), 1.5-1.9\left(\mathrm{~m}, 8 \mathrm{H}, \mathrm{CH}_{2}-\mathrm{CH}_{2}\right)$ and 190, 163, 131, 129, 115 (aromatic carbon), 67, 28, 25 (aliphatic carbon).

AEDA $_{4}$ : 9.8 (s, 2H, Ar-CHO-), 7.8 (d, 4H, ArH), 6.9 (d, 4H, ArH), $4.0\left(\mathrm{t}, 4 \mathrm{H}, \mathrm{Ar}-\mathrm{O}-\mathrm{CH}_{2}\right), 1.4-1.9\left(\mathrm{~m}, 12 \mathrm{H}, \mathrm{CH}_{2}-\mathrm{CH}_{2}\right)$ and 191, 163, 132, 129, 115 (aromatic carbon), 68, 27, 25 (aliphatic carbon).

\section{SYNTHESIS OF 4, 4' -[ALKANE-1,2-DIYLBIS(OXY-1,1-PHENYLENE)]- DIFORMATE (AEOA)}

A two-necked round-bottomed flask, equipped with a magnetic stirrer bar, thermometer, and condenser was charged with
4,4' - [alkane-1,2-diylbis(oxy)] dibenzaldehyde $(0.0370 \mathrm{~mol})$ and 3 -chloroperoxybenzoic acid $(0.1480 \mathrm{~mol})$ was added portion wise in DCM $(100 \mathrm{ml})$. The mixture was stirred at room temperature for $4 \mathrm{~h}$. A milky white suspension was formed, and the resultant reaction mixture was treated with saturated $\mathrm{NaHCO}_{3}$ solution $(50 \mathrm{ml})$. After $2 \mathrm{~h}$ stirring, the mixture was separated, the DCM layer was collected separately, and the aqueous layer was washed with DCM $(3 \times 10 \mathrm{ml})$. The organic layers were combined and washed with $10 \% \mathrm{Na}_{2} \mathrm{~S}_{2} \mathrm{O}_{3}$ solution $(3 \times 10 \mathrm{ml})$, followed by water $(3 \times 10 \mathrm{ml})$ and brine solution $(3 \times 10 \mathrm{ml})$, and finally dried over anhydrous sodium sulfate. The solvent was removed by rotary evaporation under reduced pressure to obtain a crude product. A white solid was obtained by recrystallization from warm ethanol, and the product was collected by filtration and dried in a desiccator (Scheme 1).

${ }^{1} \mathrm{HNMR}:\left(300 \mathrm{MHz}, \mathrm{CDCl}_{3}\right) \delta(\mathrm{ppm})$ and ${ }^{13} \mathrm{CNMR}: \delta(\mathrm{ppm})$ for:

$\mathrm{AEOA}_{1}: 8.3$ (s, 2H, Ar-OCHO-), 7.0 (d, 4H, ArH), 6.8 (d, 4H, $\mathrm{ArH}$ ), 4.3 (t, 4H, Ar-O-CH 2 ) and 160, 157, 143, 121, 115 (aromatic carbon), 68 (aliphatic carbon).

$\mathrm{AEOA}_{2}$ : 8.2 (s, 2H, Ar-OCHO-), 7.0 (d, 4H, ArH), 6.9 (d, 4H, $\mathrm{ArH}), 4.0\left(\mathrm{t}, 4 \mathrm{H}, \mathrm{Ar}-\mathrm{O}-\mathrm{CH}_{2}\right), 1.9\left(\mathrm{~m}, 4 \mathrm{H}, \mathrm{CH}_{2}-\mathrm{CH}_{2}\right)$ and 159, 157, 143, 121, 115 (aromatic carbon), 68, 28 (aliphatic carbon).

$\mathrm{AEOA}_{3}: 8.3$ (s, 2H, Ar-OCHO-), 7.1 (d, 4H, ArH), 7.0 (d, 4H, $\mathrm{ArH}), 4.0\left(\mathrm{t}, 4 \mathrm{H}, \mathrm{Ar}-\mathrm{O}-\mathrm{CH}_{2}\right), 1.5-1.9\left(\mathrm{~m}, 8 \mathrm{H}, \mathrm{CH}_{2}-\mathrm{CH}_{2}\right)$ and 160, 157, 143, 121, 114 (aromatic carbon), 68, 28, 26 (aliphatic carbon).

AEOA $_{4}: 8.2$ (s, 2H, Ar-OCHO-), 7.0 (d, 4H, ArH), 6.7 (d, 4H, $\mathrm{ArH}), 3.9\left(\mathrm{t}, 4 \mathrm{H}, \mathrm{Ar}-\mathrm{O}-\mathrm{CH}_{2}\right), 1.3-1.8\left(\mathrm{~m}, 12 \mathrm{H}, \mathrm{CH}_{2}-\mathrm{CH}_{2}\right)$ and 159, 157, 143, 121, 115 (aromatic carbon), 68, 29, 25 (aliphatic carbon).

\section{SYNTHESIS OF 4, 4' -[ALKANE-1,2-DIYLBIS(OXY)]DIPHENOL (AEO)}

A two-necked round-bottomed flask, equipped with a magnetic stirrer bar, thermometer, and condenser was charged with 4, 4'-[Alkane-1,2-diylbis(oxy-1,1-phenylene)]-diformate $(0.0331 \mathrm{~mol})$, sodium hydroxide $(0.1323 \mathrm{~mol})$, ethanol $(80 \mathrm{ml})$, and water $(30 \mathrm{ml})$. The mixture was stirred and heated to reflux $\left(110^{\circ} \mathrm{C}, 48 \mathrm{~h}\right)$. The reaction mixture was cooled to room temperature and neutralized with hydrochloric acid $(3 \mathrm{M})$, forming a solid that was extracted into the DCM. The DCM layers were combined, washed with water and dried over sodium sulfate; the solvent was removed by rotary evaporation under reduced pressure to obtain a crude product. A solid was obtained by recrystallization in warm ethanol; then, it was collected by filtration and dried in a hot air oven at $50^{\circ} \mathrm{C}$ for $6 \mathrm{~h}$, and stored in a desiccator (Scheme 1).

${ }^{1} \mathrm{HNMR}:\left(300 \mathrm{MHz}, \mathrm{CDCl}_{3}\right) \delta(\mathrm{ppm})$ and ${ }^{13} \mathrm{CNMR}: \delta(\mathrm{ppm})$ for:

AEO $_{1}$ : 6.7-6.6 (d, 4H, Ar-H), 4.2 (t, 4H, Ar-O-CH $)$ and 151, 150, 115, 114 (aromatic carbon), 68 (aliphatic carbon).

$\mathbf{A E O}_{2}$ : 6.7-6.6 (d, 4H, Ar-H), $4.0\left(\mathrm{t}, 4 \mathrm{H}, \mathrm{Ar}-\mathrm{O}-\mathrm{CH}_{2}\right), 1.9(\mathrm{~m}$, $4 \mathrm{H},-\mathrm{CH}_{2}-\mathrm{CH}_{2}$ ) and $151,150,115,114$ (aromatic carbon), 68, 29 (aliphatic carbon).

$\mathrm{AEO}_{3}$ : 6.7-6.5 (d, 4H, Ar-H), 3.9 (t, 4H, Ar-O-CH $), 1.4-1.8(\mathrm{~m}$, $8 \mathrm{H},-\mathrm{CH}_{2}-\mathrm{CH}_{2}$ ) and 151, 150, 115, 114 (aromatic carbon), 68, 29, 26 (aliphatic carbon). 


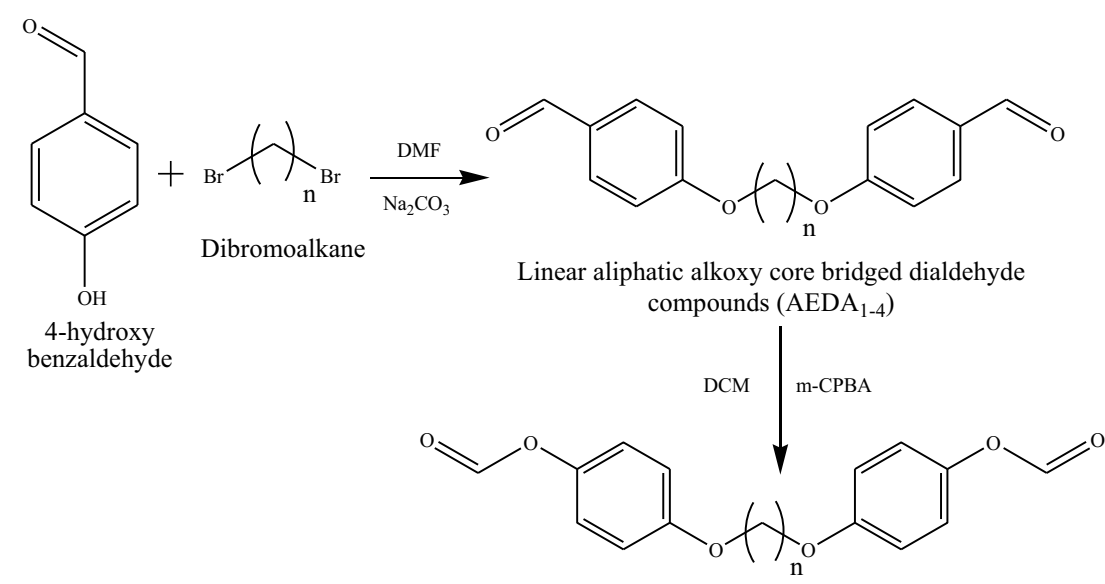

Linear aliphatic alkoxy core bridged dioxyaldehyde compounds $\left(\mathrm{AEOA}_{1-4}\right)$<smiles>CC(C)Oc1ccc(O)cc1</smiles>

Linear aliphatic alkoxy core bridged diol compounds<smiles>COc1ccc(OCC(C)(C)Oc2ccc(OC)cc2)cc1</smiles>

Linear aliphatic alkoxy core bridged cyanate esters $\left(\mathrm{AECE}_{1-4}\right)$ $\mathrm{n}=2,4,6 \& 8$

Scheme 1 | Synthesis of linear aliphatic alkoxy core bridged cyanate ester monomers (AECE1-4).

AEO $_{4}$ : 6.7-6.6 (d, 4H, Ar-H), $4.0\left(\mathrm{t}, 4 \mathrm{H}, \mathrm{Ar}-\mathrm{O}-\mathrm{CH}_{2}\right), 1.3-1.9(\mathrm{~m}$, $\left.12 \mathrm{H},-\mathrm{CH}_{2}-\mathrm{CH}_{2}\right)$ and 152, 151, 115, 114 (aromatic carbon), 68, 29, 25 (aliphatic carbon).

\section{SYNTHESIS OF 4, 4' -[ALKANE-1,2-DIYLBIS(OXY-1,1-PHENYLENE)] DICYANATE (AECE)}

A three-necked round-bottomed flask, equipped with a magnetic stirrer bar, a dropping funnel, and a drying tube, was charged with 4, 4' -[Alkane-1,2-diylbis(oxy)]diphenol $(0.0203 \mathrm{~mol})$ and cyanogen bromide $(0.0447 \mathrm{~mol})$. The reactants were dissolved in acetone $(75 \mathrm{ml})$, and the solution was cooled to below $-5^{\circ} \mathrm{C}$ in a salt ice bath. Triethylamine $(0.0507 \mathrm{~mol})$ was added dropwise via the dropping funnel to the reaction mixture and was maintained below $-5^{\circ} \mathrm{C}$. After the addition of triethylamine, the reaction mixture was left stirring for $1 \mathrm{~h}$ and then allowed to $25^{\circ} \mathrm{C}$. The mixture was washed by fast stirring with water to remove triethylamine hydrobromide (byproduct) and the remaining precipitate was filtered and washed with water. A light white solid product was obtained by recrystallization from 1:1 mixture of ethanol and water (Scheme 1).

\section{$\mathrm{AECE}_{1}$ :}

FT-IR: $\left(\mathrm{KBr}, \mathrm{cm}^{-1}\right)$ : 2937(symmetric stretching), 2862(asymmetric stretching) for $\mathrm{CH}_{2}$ aliphatic Stretching, 2277, 2229 (-OCN stretching), 1243( $\left.\mathrm{Ar}-\mathrm{O}-\mathrm{CH}_{2}\right)$.

${ }^{1} \mathrm{HNMR}:\left(300 \mathrm{MHz}, \mathrm{CDCl}_{3}\right) \delta(\mathrm{ppm}): 7.2$ (d, $\left.4 \mathrm{H}, \mathrm{ArH}\right), 7.0(\mathrm{~d}$, $4 \mathrm{H}, \mathrm{ArH}), 4.3$ (t, $\left.4 \mathrm{H}, \mathrm{Ar}-\mathrm{O}-\mathrm{CH}_{2}\right)$.

${ }^{13}$ CNMR: $\delta$ (ppm): 157,146,116,115, 109 (aromatic carbon), 68(aliphatic carbon).

$\mathrm{AECE}_{2}$ :

FT-IR: $\left(\mathrm{KBr}, \mathrm{cm}^{-1}\right.$ ): 2940 (symmetric stretching), 2858 (asymmetric stretching) for $\mathrm{CH}_{2}$ aliphatic Stretching and 2278, 2233 (-OCN stretching), 1249 (Ar-O-CH 2$)$.

${ }^{1} \mathrm{HNMR}:\left(300 \mathrm{MHz}, \mathrm{CDCl}_{3}\right) \delta(\mathrm{ppm}): 7.2(\mathrm{~d}, 4 \mathrm{H}, \mathrm{ArH}), 6.9(\mathrm{~d}$, $4 \mathrm{H}, \mathrm{ArH}), 4.0$ ( $\left.\mathrm{t}, 4 \mathrm{H}, \mathrm{Ar}-\mathrm{O}-\mathrm{CH}_{2}\right), 2.0\left(\mathrm{~m}, 4 \mathrm{H},-\mathrm{CH}_{2}-\right)$. 
${ }^{13}$ CNMR: $\delta$ (ppm): 157,146,116, 115,109 (aromatic carbon), 68, 25 (aliphatic carbon).

$\mathrm{AECE}_{3}$ :

FT-IR: $\left(\mathrm{KBr}, \mathrm{cm}^{-1}\right)$ : 2941 (symmetric stretching), 2862 (asymmetric stretching), 2272, 2230 (-OCN stretching) for $\mathrm{CH}_{2}$ aliphatic Stretching, $1245\left(\mathrm{Ar}-\mathrm{O}-\mathrm{CH}_{2}\right)$.

${ }^{1} \mathrm{HNMR}:\left(300 \mathrm{MHz}, \mathrm{CDCl}_{3}\right) \delta(\mathrm{ppm}): 7.2(\mathrm{~d}, 4 \mathrm{H}, \mathrm{ArH}), 6.9(\mathrm{~d}$, $4 \mathrm{H}, \mathrm{ArH}), 4.0\left(\mathrm{t}, 4 \mathrm{H}, \mathrm{Ar}-\mathrm{O}-\mathrm{CH}_{2}\right), 1.8\left(\mathrm{~m}, 4 \mathrm{H},-\mathrm{CH}_{2}-\right), 1.5(\mathrm{~m}$, $\left.4 \mathrm{H},-\mathrm{CH}_{2}-\right)$.

${ }^{13}$ CNMR: $\delta$ (ppm): 157,146,116,115,109 (aromatic carbon), 68, 29, 25, (aliphatic carbon).

$\mathrm{AECE}_{4}$ :

FT-IR: (KBr, cm ${ }^{-1}$ ): 2937(symmetric stretching), 2856 (asymmetric stretching) for $\mathrm{CH}_{2}$ aliphatic Stretching, 2278, 2230 (-OCN stretching), $1242\left(\mathrm{Ar}-\mathrm{O}-\mathrm{CH}_{2}\right)$.

${ }^{1} \mathrm{HNMR}:\left(300 \mathrm{MHz}, \mathrm{CDCl}_{3}\right) \delta(\mathrm{ppm}): 7.2(\mathrm{~d}, 4 \mathrm{H}, \mathrm{ArH}), 6.8$ $(\mathrm{d}, 4 \mathrm{H}, \mathrm{ArH}), 4.0\left(\mathrm{t}, 4 \mathrm{H}, \mathrm{Ar}-\mathrm{O}-\mathrm{CH}_{2}\right), 1.9-1.7\left(\mathrm{~m}, 8 \mathrm{H},-\mathrm{CH}_{2}-\right)$, $1.4\left(\mathrm{~m}, 4 \mathrm{H},-\mathrm{CH}_{2}-\right)$.

${ }^{13}$ CNMR: $\delta$ (ppm): 156,145,115,114, 107 (aromatic carbon), 67, 27, 21 (aliphatic carbon).

\section{PREPARATION OF POSS-CE NANOCOMPOSITES}

$2 \mathrm{~g}$ of CE monomer was first dissolved in $10 \mathrm{ml}$ of 1,4-dioxane. The desired amount of OG-POSS $(0,5$, and $10 \mathrm{wt} \%)$ was dissolved in minimum amount of 1,4-dioxane. The homogeneous mixture was subjected to sonic waves in a sonicator for effective collision and distribution at $25^{\circ} \mathrm{C}$ for $1 \mathrm{~h}$. The blends were cast on a glass plate and pre-treated with dichlorodimethylsilane. After drying at $60^{\circ} \mathrm{C}$ for $5 \mathrm{~h}$, the blended samples were cured at 100 , 150,200 for $1 \mathrm{~h}$ each and $250^{\circ} \mathrm{C}$ for $2 \mathrm{~h}$ in an air oven. The red brown colored POSS reinforced CE (POSS-AECE) nanocomposites were obtained with thicknesses ranging from 0.3 to $0.5 \mathrm{~mm}$ (Scheme 2).

\section{CHARACTERIZATION}

FT-IR spectra were recorded on a Perkin Elmer $6 \times$ FT-IR spectrometer. About $100 \mathrm{mg}$ of optical-grade $\mathrm{KBr}$ was ground with sufficient quantity of the solid sample to make $1 \mathrm{wt} \%$ mixture for making $\mathrm{KBr}$ pellets. All ${ }^{1} \mathrm{H}$ and ${ }^{13} \mathrm{C}$ NMR analyses were done in $\mathrm{CDCl}_{3}$ and recorded on a Bruker 300 and Bruker 500 spectrometer.

The calorimetric analysis was performed on a Netzsch DSC200 differential scanning calorimeter. Measurements were performed under a continuous flow of nitrogen $(20 \mathrm{~mL} / \mathrm{min})$. All the samples (about $10 \mathrm{mg}$ in weight) were heated from ambient temperature to $300^{\circ} \mathrm{C}$ and the thermograms were recorded at a heating rate of $10^{\circ} \mathrm{C} / \mathrm{min}$. Thermo gravimetric analysis (TGA) was performed on a Netzsch STA 409 thermo gravimetric analyzer. The samples (about $10 \mathrm{mg}$ ) were heated from ambient temperature to $700^{\circ} \mathrm{C}$ under a continuous flow of nitrogen $(20 \mathrm{~mL} / \mathrm{min})$, at $10^{\circ} \mathrm{C} / \mathrm{min}$.

The dielectric constant of the neat AECE and the POSS-AECE systems were determined with the help of impedance analyser (Solartron impedance/gain phase analyser 1260) at room temperature using platinum $(\mathrm{Pt})$ electrode at a frequency range of $1 \mathrm{MHz}$. This experiment was repeated four times at the same conditions.
A JEOL JSM-6360 field emission Scanning Electron Microscope (SEM) was used to record the SEM pictures and the samples were prepared by coating gold on their surface for SEM measurements. A JEOL JEM-3010 analytical transmission electron microscope, operating at $300 \mathrm{kV}$ with a measured point-to-point resolution of $0.23 \mathrm{~nm}$ has been used to capture the micrograph of the composites. TEM samples were prepared by dissolving polymers in DMF mounted on carbon-coated $\mathrm{Cu}$ TEM grids and dried $24 \mathrm{~h}$ at RT to form a film in $<100 \mathrm{~nm}$ size.

Water contact angle measurements were taken using a Ramehart Inc. goniometer. Contact angle measurements were taken with $5 \mu \mathrm{l}$ of water and DI used as probe liquid.

\section{RESULTS AND DISCUSSION}

The flexible linear aliphatic alkoxy core bridged bisphenol CE (Scheme 1) was synthesized with good yield, in four steps; in the first step, the synthesis of linear dialdehyde by o-arylation, using the respective dibromoalkane $\left[\left(\mathrm{CH}_{2}\right)_{n} n=2,4,6\right.$, and 8] and 4-hydroxy benzaldehyde in the presence of anhydrous sodium carbonate in DMF was done. In the second step, the linear dialdehyde was oxidized using MCPBA to get linear oxydialdehyde, and in the third step the hydrolysis of linear oxydialdehyde was carried out to get the respective ether linked diols. Finally, during the fourth step the cyanogenation of diols was done to get the respective linear ether linked cyanate esters. The molecular structure of all monomers was confirmed by the FT-IR (Figure 1). From FTIR spectra, the appearances of peak at $2229-2277 \mathrm{~cm}^{-1}$ for OCN and 2862, $2937 \mathrm{~cm}^{-1}$ correspond to symmetric and asymmetric stretching, respectively for aliphatic $\mathrm{CH}_{2}$ group. The ${ }^{1} \mathrm{H}$ and ${ }^{13} \mathrm{CNMR}$ (Figures $\mathrm{S} 1-\mathrm{S} 8$ present in supporting information) further confirms the structure of AECE monomers.

One of the key disadvantages of conventional CE resins is their poor curing characteristic; that is, normally CE should be cured at a higher curing temperature to obtain the desired performance. Generally, higher curing temperatures result in higher internal stress and more defects in the cured resin, thereby decreasing the overall properties of the resultant resin. The curing behavior of the linear ether linked CE monomers was studied by the DSC analysis. The DSC thermogram of linear ether linked CE monomers is shown in Figure 2. The DSC thermogram shows a single exothermic peak which is attributed to the cyclotrimerization of CE. The onset of the curing of CE monomers commences at a lower temperature, with an increase in the chain length of the monomer. The $-\Delta \mathrm{H}$ polymerization enthalpy values for $\mathrm{AECE}_{1-4}$ monomers are in the range between $567 \mathrm{~mJ} / \mathrm{mg}$ and $355 \mathrm{~mJ} / \mathrm{mg}$. In the case of $\mathrm{AECE}_{4}$ monomer, the onset and the peak top temperatures are lower and the $\mathrm{AECE}_{1}$ monomer shows a higher onset and peak top temperature. In the case of the other monomers $\left(\mathrm{AECE}_{2}, \mathrm{AECE}_{3}\right)$, the onset and peak top temperatures lie in between these two extremes, which are lower than that of conventional CE monomer (BADy) and the values are presented in Table 1. This can be explained by the presence of the electron donating ether linkage; the aliphatic ether chain length increases and may lead to an increase in the electron density, which decreases the curing temperature (Vengatesan et al., 2013).

The POSS-AECE nanocomposites (Scheme 2) were prepared by the incorporation of varying weight percentages of OG-POSS 


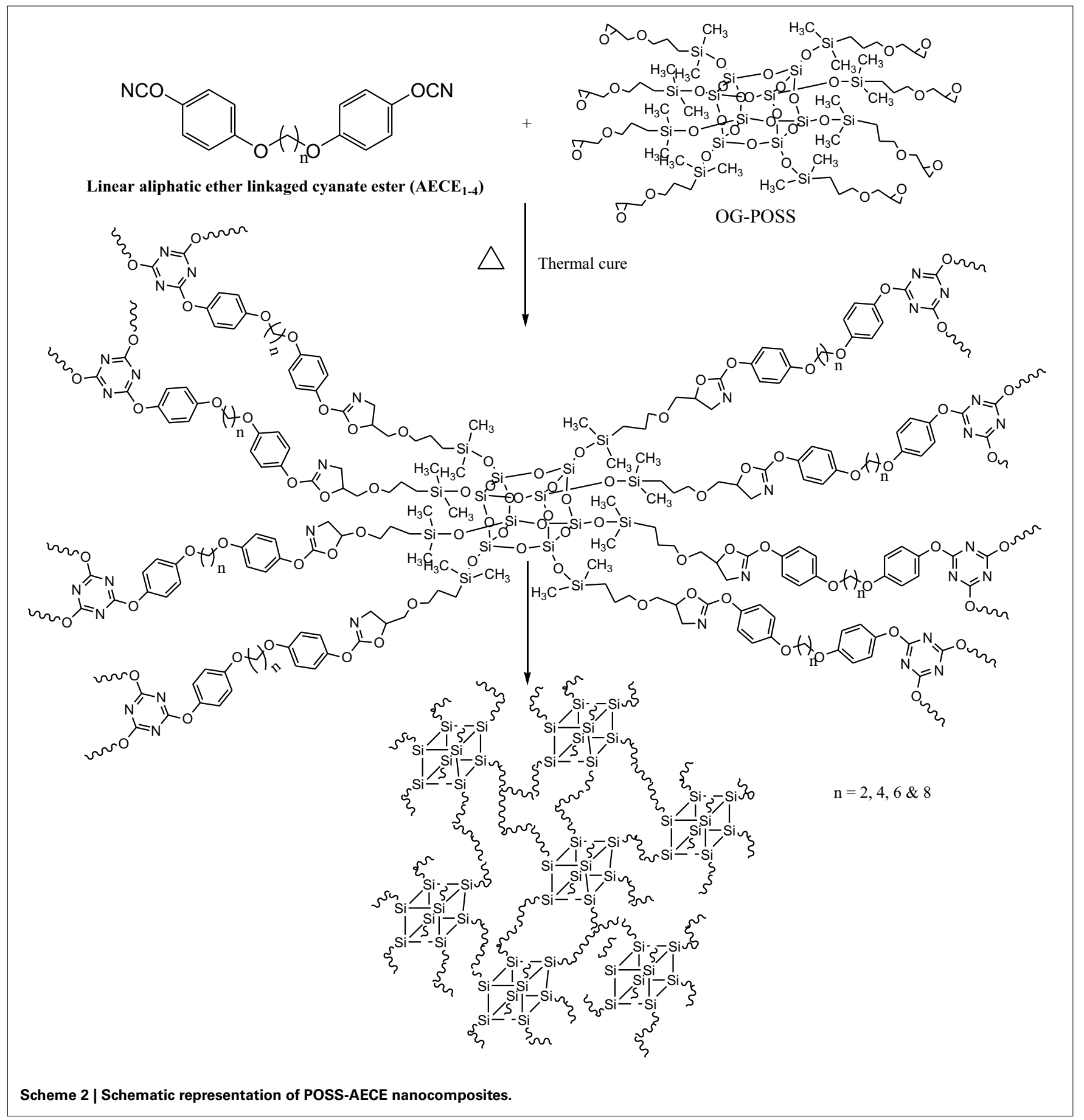

( 5 and 10 wt \%) in to AECE resin. Figure 3 shows the FTIR spectra of varying combinations of POSS-AECE nanocomposites. The disappearance of the bands at $2237 \mathrm{~cm}^{-1}$ (the -OCN group of the CE) and $914 \mathrm{~cm}^{-1}$ (the glycidyl group of OG-POSS), and the appearance of a band at $1635 \mathrm{~cm}^{-1}$ corresponding to an oxazoline linkage, confirms the occurrence of a reaction between the OCN group of CE and glycidyl group of OG-POSS. The symmetric and asymmetric stretching frequencies of the $-\mathrm{CH}_{2}$ group appeared at 2924 and $2868 \mathrm{~cm}^{-1}$, respectively. The presence of a band at $1098 \mathrm{~cm}^{-1}$ due to $\mathrm{Si}-\mathrm{O}-\mathrm{Si}$ confirms the presence of a silica network in the hybrid.

The glass transition temperature (Netzsch DSC-200) of the neat AECEs and POSS-AECE nanocomposites are shown in Table 2. The POSS-AECE nanocomposites exhibit increased $\mathrm{Tg}$ compared to that of neat AECE and this may be explained by the following reasons. The incorporation of POSS increases the crosslinking density of the resulting nanocomposites, and increases the rigidity of the nanocomposites system. Also the increase in the 


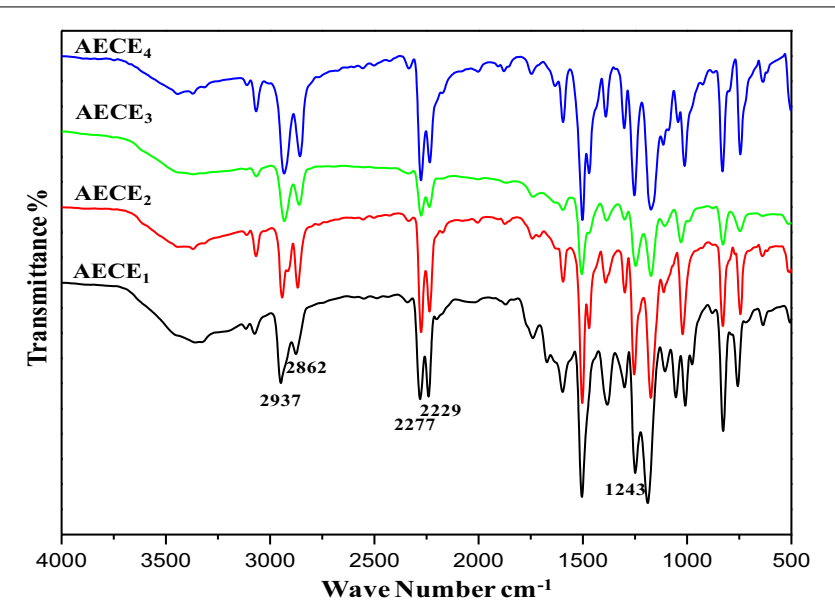

Figure 1 | FT-IR spectra of linear aliphatic alkoxy core bridged cyanate esters.

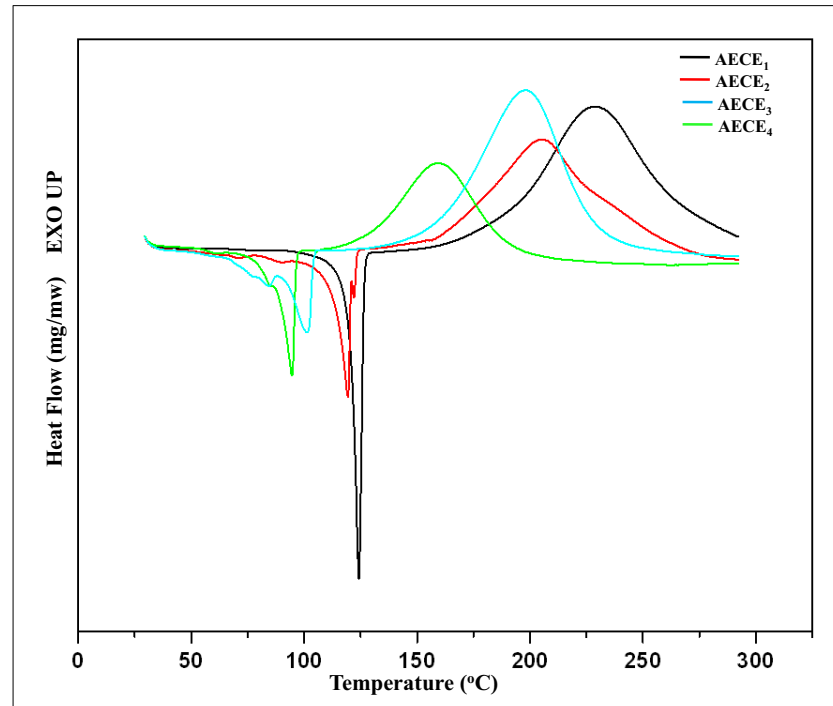

Figure 2 | Curing behaviors of cyanate ester monomers $\left(\mathrm{AECE}_{1-4}\right)$.

Table 1 | Curing studies of AECE monomers.

\begin{tabular}{lllll}
\hline Sample & $\mathbf{T}_{\mathbf{i}}\left({ }^{\circ} \mathbf{C}\right)$ & $\mathbf{T}_{\mathbf{p}}\left({ }^{\circ} \mathbf{C}\right)$ & $\mathbf{T}_{\mathbf{f}}\left({ }^{\circ} \mathbf{C}\right)$ & $\mathbf{\Delta} \mathbf{H}$ polymerization $\mathbf{~} \mathbf{J} / \mathbf{m g}$ \\
\hline AECE $_{1}$ & 175 & 228 & 263 & 569 \\
AECE $_{2}$ & 163 & 205 & 251 & 512 \\
AECE $_{3}$ & 144 & 197 & 230 & 446 \\
AECE $_{4}$ & 120 & 161 & 186 & 355
\end{tabular}

$T_{i}$, initial polymerization temperature; $T_{p}$, peak curing temperature of the monomers; $T_{f}$, final polymerization temperature.

values of $\mathrm{Tg}$, is due to the restricted segmental motion imparted by the polymer chain with chemical interaction between the polymeric chain and POSS. The incorporation of POSS creates porosities (free volume) in the nanocomposites; this effect has, however, been counteracted by an increase in the cross-linking

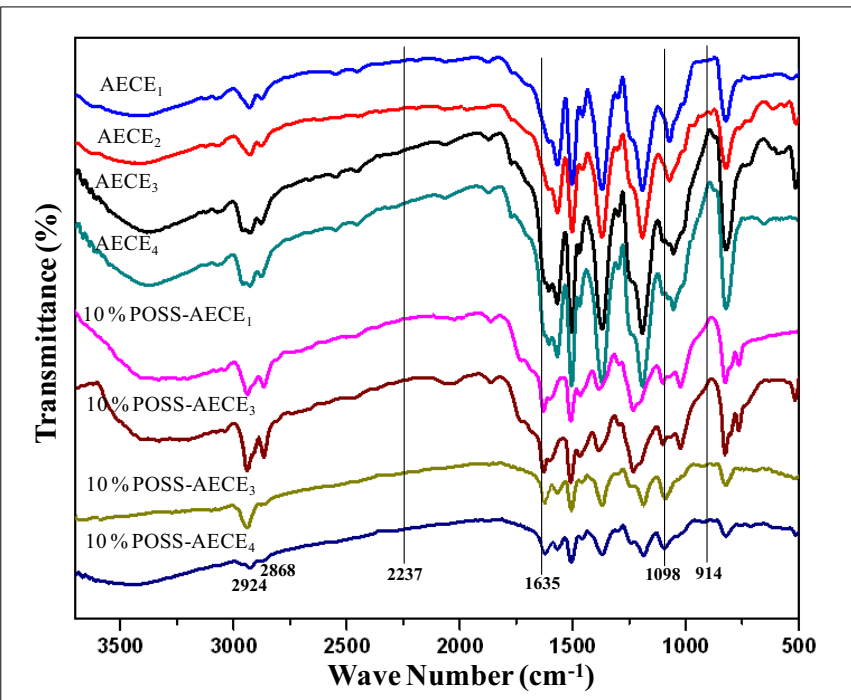

Figure 3 | FT-IR spectra of POSS-AECE nanocomposites.

Table 2 | Thermal and dielectric properties of neat AECE and POSS-AECE nanocomposites.

\begin{tabular}{|c|c|c|c|c|c|}
\hline Experiment & $\mathrm{T}_{\mathrm{g}}\left({ }^{\circ} \mathrm{C}\right)$ & $\begin{array}{l}10 \% \\
\text { weight } \\
\text { loss }\left({ }^{\circ} \mathrm{C}\right)\end{array}$ & $\begin{array}{l}50 \% \\
\text { weight } \\
\text { loss }\left({ }^{\circ} \mathrm{C}\right)\end{array}$ & $\begin{array}{l}\text { Char } \\
\text { yield (at } \\
\left.900^{\circ} \mathrm{C}\right) \\
(\%)\end{array}$ & $\begin{array}{l}\text { Dielectric } \\
\text { constant } \\
(\varepsilon)\end{array}$ \\
\hline $\mathrm{AECE}_{1}$ & 220 & 336 & 417 & 20.1 & 4.2 \\
\hline $5 \%$ POSS-AECE 1 & 228 & 351 & 425 & 23.5 & 3.6 \\
\hline $10 \%$ POSS-AECE 1 & 235 & 375 & 430 & 27.2 & 3.1 \\
\hline $\mathrm{AECE}_{2}$ & 217 & 330 & 423 & 16.2 & 3.8 \\
\hline $5 \%$ POSS-AECE 2 & 222 & 329 & 427 & 19.0 & 3.5 \\
\hline $10 \%$ POSS-AECE 2 & 230 & 331 & 429 & 21.4 & 2.9 \\
\hline $\mathrm{AECE}_{3}$ & 210 & 328 & 410 & 10.2 & 3.6 \\
\hline $5 \%$ POSS-AECE $_{3}$ & 223 & 325 & 420 & 15.1 & 3.0 \\
\hline $10 \%$ POSS-AECE 3 & 228 & 321 & 427 & 19.4 & 2.6 \\
\hline $\mathrm{AECE}_{4}$ & 203 & 336 & 420 & 5.1 & 3.2 \\
\hline $5 \%$ POSS-AECE $_{4}$ & 211 & 338 & 425 & 14.8 & 2.9 \\
\hline $10 \%$ POSS-AECE 4 & 221 & 341 & 440 & 16.0 & 2.4 \\
\hline
\end{tabular}

density. Among the POSS-AECE systems the AECE 1 based system possesses higher values of $\mathrm{Tg}$ and the $\mathrm{AECE}_{4}$ based system exhibits the lowest value of $\mathrm{Tg}$. The values of the other systems are lie in between these two systems. The Tg value of POSS-AECE decreased linearly with an increasing chain length. The flexible aliphatic backbone influences the glass transition temperature of the hybrid systems by increasing the internal rotations and thermal motion of the polymer chains, which in turn decreases the values of $\mathrm{Tg}$.

Thermo gravimetric analysis (Netzsch STA 409) was used to ascertain the thermal stability of neat AECE and OG-POSS reinforced AECE nanocomposites, and the data obtained from the TGA are presented in Figure 4 and Table 2. The POSS-AECE nanocomposites exhibited higher thermal stability and higher 

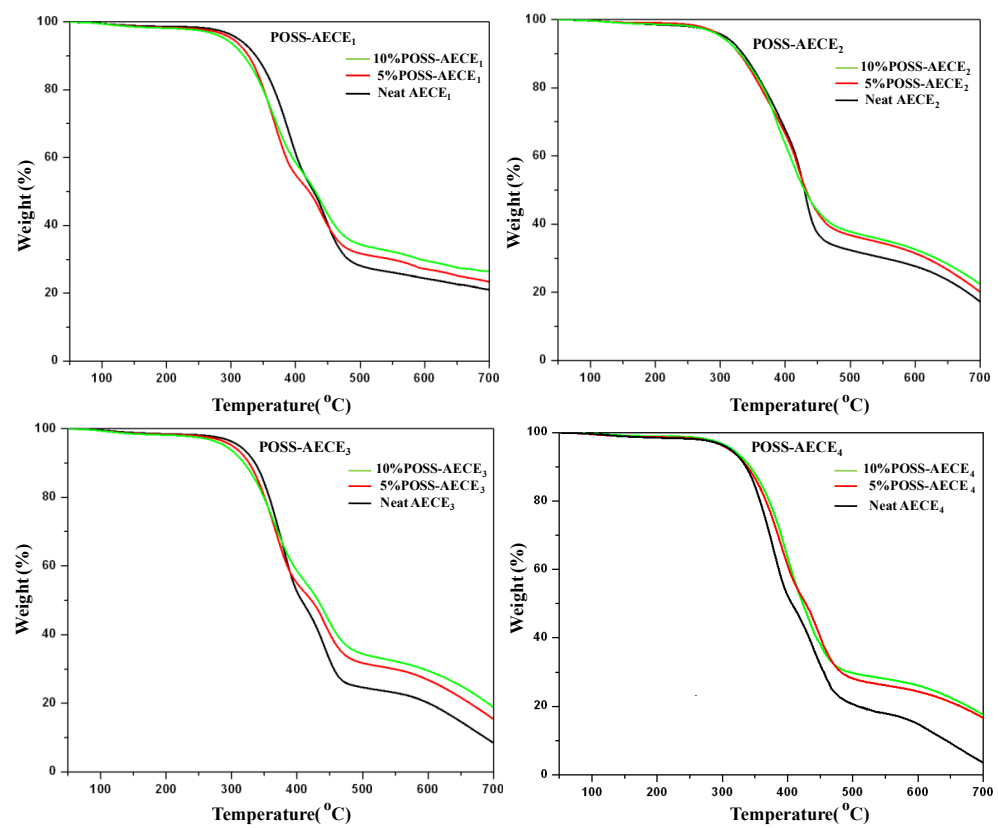

Figure 4 | TGA thermogram of POSS-AECE nanocomposites.

char yield than those of neat AECE resin. Generally, the improvement in the thermal stability of hybrid composites is related to the degree of the interaction between the polymer matrix and the inorganic phase, and to the fact that the inorganic component has an inherently good thermal stability. In addition, the presence of the rigid cubic siloxane and the partial ionic nature of the inorganic Si-O-Si skeleton contributed to the thermal stability of the hybrid nanocomposites. The POSS core gave an additional heat capacity which stabilizes the materials against the thermal decomposition. The loss of organic materials from the segmental decomposition through gaseous fragments could be reduced by the well dispersed POSS core in the AECE matrix. Further, it was also observed that the incorporation of OG-POSS into the AECE resin reduced both the volatile decomposition and the polymer flammability. Further, when POSS degrades, it leaves an inert silica layer which can form a protective layer on the surface of the material preventing further oxidation of the inner part of the matrix. Among the four nanocomposites $\left(\mathrm{AECE}_{1-4}\right)$, the $\mathrm{AECE}_{1}$ based POSS incorporated nanocomposites possesses higher thermal stability, while that of $\mathrm{AECE}_{4}$ nanocomposites exhibits the lowest thermal stability due to the higher chain length, which induces free rotational movement.

A lower dielectric constant is one of the most desirable properties for next generation microelectronic devices. Table 1 gives the values of the dielectric constant (Solartron impedance/gain phase analyzer 1260) of POSS-AECE nanocomposites with varying percentage of POSS concentration. The dielectric constant of the hybrid decreases with an increase in the amount of POSS. The reduction in the value of dielectric constant of POSS-AECE hybrids is due to the creation of pores and enhanced free volume contributed by the rigid and bulky core structured POSS molecule
(Wu et al., 2007). The less polar nature of Si-O-Si linkage and aliphatic flexible group in the POSS-AECE system reduces the value of dielectric constant. The signal propagation delay time of the integrated circuits is proportional to the square root of the dielectric constant of the matrix, and the signal propagation loss is proportional to the square root of the dielectric constant and dissipation factor of the matrix. Thus, a material with a low dielectric constant and low dissipation factor will reduce the signal propagation delay time and the signal propagation loss (Nagendiran et al., 2010), and can be used to improve the effective and efficient functioning of electronic instruments. Among the POSS-AECE systems studied, the $\mathrm{AECE}_{4}$ based system possesses the lowest value of dielectric constant and $\mathrm{AECE}_{1}$ based system exhibits the highest value of dielectric constant. The values of the other two systems are lie in between these two extremes. This may be explained by the presence of higher aliphatic chain which reduces the polarity and enhances the free rotation of the molecules.

The SEM (JEOL JSM-6360) is used to investigate the morphology of neat CE and OG-POSS reinforced AECE nanocomposites. Figure 5 shows the SEM micrograph of neat AECE and POSSAECE nanocomposites. The SEM images indicated the smooth and homogeneous morphology of the neat AECE and POSSAECE systems. The POSS incorporated system exhibited a featureless morphology and no discernable phase separation was observed. This indicates the good miscibility (compatibility) of POSS with AECE and also confirms the molecular level dispersion of POSS in the AECE nanocomposites. The homogeneous morphology could be ascribed to the formation of covalent bonding between POSS and the CE. This was further confirmed by the TEM analysis. 
Figures 6A-D represents the TEM micrographs (JEOL JEM3010) of $10 \mathrm{wt} \%$ of OG-POSS reinforced AECE nanocomposites. It shows the homogeneous morphology, and no localized domains were observed. Only a few darker points were observed at approximately about $50 \mathrm{~nm}$ in the polymer matrix, which represents the dispersion of the OG-POSS in the AECE matrix. This observation indicates that the nanocomposite is a material with a
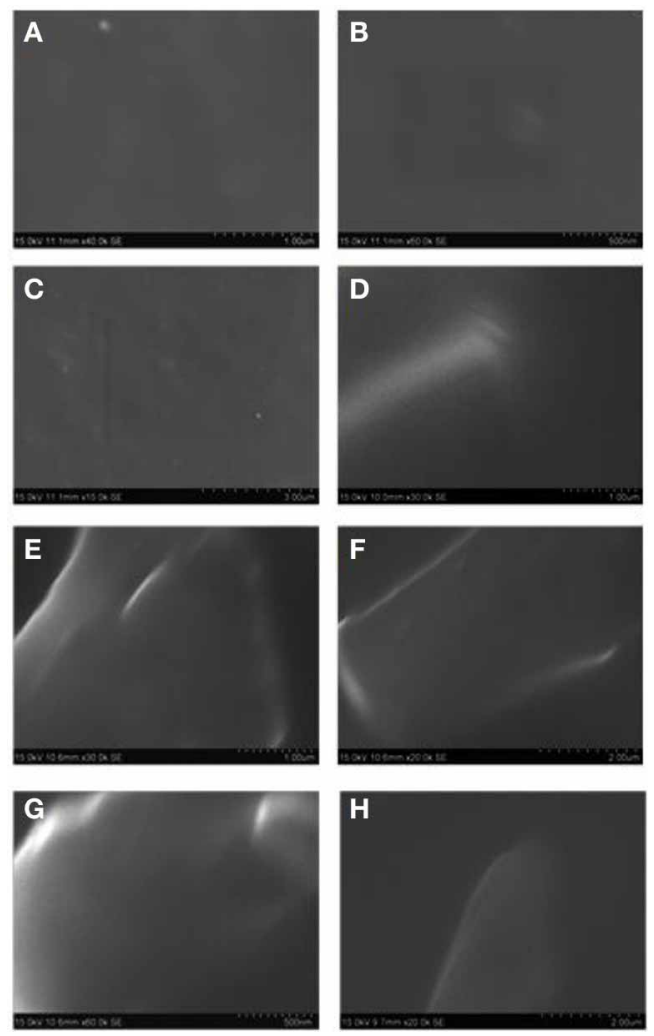

Figure 5 | SEM micrograph of (A). Neat $A E C E_{1}$, (B). 10\% POSS-AECE 1 , (C). AECE 2 , (D). 10\% POSS-AECE2, (E). Neat AECE 3 , (F). 10\% POSS-AECE 3 , (G). Neat AECE 4 and (H). 10\% POSS-AECE 4 .
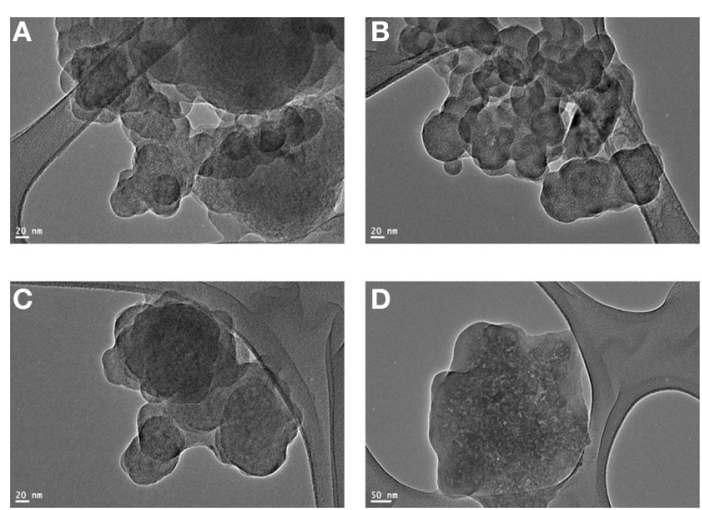

Figure 6 | TEM image of (A). 10\% POSS-AECE 1 , (B). 10\% POSS-AECE 2 , (C). $10 \%$ POSS-AECE $_{3}$ and (D). $10 \%$ POSS-AECE $_{4}$. particle size of the dispersed phase having at least one dimension of less than $100 \mathrm{~nm}$ (Komarneni, 1992). Thus, the POSS moieties are well dispersed at a nanometer scale in the AECE matrix to form a POSS-AECE network.

The hydrophobicity of the neat AECEs and POSS incorporated POSS-AECEs was determined from the contact angle measurements using the goniometer (GBX, France). Contact angle measurements were taken with water and diiodomethane, to determine how POSS influences the hydrophobic nature of the CE matrix. The values of contact angle of neat AECE and POSS incorporated AECEs are presented in Table 3. With an incorporation of 5 and $10 \mathrm{wt} \%$ OG-POSS, the values of the contact angles increased, which indicates that the presence of POSS in the AECE matrix improves the hydrophobicity of the nanocomposite surfaces. The increase in hydrophobicity is mainly ascribed to the covalent interaction between POSS and AECEs, and also the less polar nature of the Si-O-Si linkage in the POSS, which leads to an increase in the hydrophobic nature of the AECE matrices (Tang and Lewin, 2009; Wang et al., 2011). Among the four systems the $\mathrm{AECE}_{4}$ based POSS incorporated system possesses the highest value of water contact angle, and the AECE 1 based POSS incorporated system exhibits the lowest value of the water contact angle. This may be explained due to the higher aliphatic flexible linear alkoxy chain that reduces the polarity, and increases the hydrophobic nature of the resulting hybrid system compared to that of the other three systems.

Two approaches are employed to calculate the surface energy of a solid material: the geometric mean method (Wang et al., 2011), and three liquid method (Xiang and Chung, 2001). These two methods are the most widely used for surface energy analyses.

In this work we used the geometric mean method estimates the surface energy of a solid $\left(\gamma_{S}\right)$ as follows:

$$
\operatorname{Cos} \theta=2 / \gamma_{L}\left[\left(\gamma_{L}^{d} \cdot \gamma_{S}^{d}\right)^{1 / 2}+\left(\gamma_{L}^{p} \cdot \gamma_{S}^{p}\right)^{1 / 2}\right]-1
$$

Table 3 | Contact angle and surface free energy of neat AECE and POSS-AECE nanocomposites.

\begin{tabular}{|c|c|c|c|c|c|}
\hline \multirow[t]{2}{*}{ Experiments } & \multicolumn{2}{|c|}{ Contac angle $(\theta)$} & \multicolumn{3}{|c|}{ Surface free energy } \\
\hline & Water & Diiodomethane & $\gamma^{\mathrm{d}}$ & $\gamma^{\mathbf{p}}$ & $\Gamma$ \\
\hline $\mathrm{AECE}_{1}$ & 85 & 48 & 35.4 & 2.7 & 38.1 \\
\hline $5 \%$ POSS-AECE 1 & 94 & 60 & 28.6 & 1.6 & 30.1 \\
\hline $10 \%$ POSS-AECE 1 & 103 & 67 & 24.6 & 0.5 & 25.1 \\
\hline $\mathrm{AECE}_{2}$ & 88 & 52 & 33.2 & 2.0 & 35.2 \\
\hline $5 \%$ POSS-AECE 2 & 98 & 63 & 26.8 & 1.0 & 27.8 \\
\hline $10 \%$ POSS-AECE 2 & 107 & 70 & 22.9 & 0.2 & 23.1 \\
\hline $\mathrm{AECE}_{3}$ & 93 & 58 & 29.7 & 1.6 & 31.3 \\
\hline $5 \%$ POSS-AECE $_{3}$ & 104 & 68 & 24.0 & 0.4 & 24.4 \\
\hline $10 \%$ POSS-AECE 3 & 112 & 75 & 20.1 & 0.1 & 20.2 \\
\hline $\mathrm{AECE}_{4}$ & 98 & 59 & 29.2 & 0.9 & 30.0 \\
\hline $5 \%$ POSS-AECE $_{4}$ & 109 & 71 & 22.3 & 0.1 & 22.4 \\
\hline $10 \%$ POSS-AECE 4 & 121 & 77 & 19.1 & 0.1 & 19.2 \\
\hline
\end{tabular}


where $\gamma_{L}, \gamma_{L}^{d}$, and $\gamma_{L}^{p}$ are the surface energies of the test liquid and $\gamma_{S}^{d}$ and $\gamma_{S}^{p}$ can be calculated from the measured value of contact angles $(\theta)$. The $d$ refers to the London dispersion forces and $p$ refers to the polar forces, including all the interactions established between the solid and liquid, such as Keesom dipole-dipole, Debye dipole-induced dipole, and hydrogen bonding, etc. A low surface free energy is important in many practical applications. Considerable effort has been focused on the development of nonwettable, low surface free energy polymeric materials with film forming characteristics. The thrust for those activities is provided by commercial applications in aerospace, lithography, clothing, wetting, dyeing, intergraded sensors, and protection against biological and other fouling. In general, amorphous, comb like polymers possessing a flexible linear backbone onto which sidechains with low intermolecular interactions are attached, exhibit low surface free energy values (Tsibouklis and Nevell, 2003).

The surface free energy values of neat AECEs and POSSAECEs are listed in Table 3. From the Table it can be seen that the surface free energy value decreases with an increase in the percentage content of POSS incorporated in to AECE matrices. This is due to the flexible aliphatic alkoxy core that reduces the polarity of the system; also, the less polar Si-OSi linkage in the POSS covalently incorporated into the AECE matrix, which reduces the value of the surface free energy of the resulting composite system (Tang and Lewin, 2009; Wang et al., 2011). Among the four systems, the $\mathrm{AECE}_{4}$ based system exhibited the lowest values of surface free energy when compared to that of other three hybrid systems and this is

\section{REFERENCES}

Chandramohan, A., Devaraju, S., Vengatesan, M. R., and Alagar, M. (2012). Octakis(dimethylsiloxy propylglycidylether) silsesquioxane (OG-POSS) reinforced 1, 1-bis(3-methyl-4-hydroxymethyl) cyclohexane based polybenzoxazine nanocomposites. J. Polym. Res. 19, 9903-9912. doi: 10.1007/s10965-012-9903-8

Cordes, D. B., Lickiss, P. D., and Rataboul, F. (2010). Recent developments in the chemistry of cubic polyhedral oligosilsesquioxanes. Chem. Rev. 110, 2081-2173. doi: 10.1021/ cr900201r

Dang, Z. M., Yu, Y. F., Xu, H. P., and Bai, J. (2008). Study on microstructure and dielectric property of the BaTiO3/epoxy resin composites. J. Compos. Sci. Technol. 68, 171-177. doi: 10.1016/j.compscitech.2007. 05.021

Devaraju, S., Venkatesan, M. R., and Alagar, M. (2011). Studies on thermal and dielectric properties of ether linked cyclohexyl diamine (ELCD)-based polyimide Pnanocomposites, OSS (POSS-PI).
High Perform. Polym. 23, 99-111. doi: $10.1177 / 0954008310391825$

Devaraju, S., Venkatesan, M. R., Selvi, M., Ashok Kumar, A., and Alagar, M. (2012). Synthesis and characterization of bisphenol-A ether diamine-based polyimide POSS nanocomposites for low $\mathrm{K}$ dielectric and flame-retardant applications. High Perform. Polym. 24, 84-96. doi: 10.1177/0954008311433606

Devaux, E., Rochery, M., and Bourbigot, S. (2002). Polyurethane/clay and polyurethane/POSS nanocomposites as flame retarded coating for polyester and cotton fabrics. Fire Mater. 26, 149-154. doi: $10.1002 /$ fam.792

Fang, T., and Shimp, D. A. (1995). Polycyanate esters: science and applications. Prog. Polym. Sci. 20, 61-118. doi: 10.1016/0079-6700(94)E0006-M

Gonzalez, R. I., Phillips, S. H., and Hoflund, G. B. (2000). In situ oxygen-atom erosion study of polyhedral oligomeric silsesquioxane-siloxane copolymer. J. Spacecraft Rockets 37, 463-467. doi: $10.2514 / 2.3606$ due to the presence of higher aliphatic flexible linear alkoxy chain which reduces the polarity of the resulting composite systems.

\section{CONCLUSION}

A new series of linear aliphatic alkoxy core bridged bisphenol CE based POSS-AECE hybrid nanocomposites have been developed. The formation of nanocomposites was confirmed by the FT-IR. Data from the thermal analysis indicate that POSS-AECE nanocomposites exhibit excellent thermal properties, such as higher Tg, thermal stability and higher char yield, than those of neat AECE. The values of the dielectric constant $(\hat{\epsilon})$ of the POSS incorporated AECE are lower than those of neat AECE. From the contact angle studies it is inferred that the POSS incorporated AECE system possesses better hydrophobicity and lower surface free energy, when compared to those of neat AECE.

\section{ACKNOWLEDGMENTS}

The authors thank BRNS, G. No: 2012/37C/9/BRNS, Mumbai, Govt. of India., for the financial support and Dr. Manmohan Kumar, Senior Scientific Officer, BARC, Mumbai. Authors also thank the Department of Mechanical Engineering, Anna University, for providing facility for SEM analysis and PSG College of Technology for TEM analysis.

\section{SUPPLEMENTARY MATERIAL}

The Supplementary Material for this article can be found online at: http://www.frontiersin.org/journal/10.3389/fchem.2013.00019/ abstract
Jang, J., Nam, S., Chung, D. S., Kim, S. H., Yun, W. M., and Park, C. E. (2010). High Tg cyclic olefin copolymer gate dielectrics for N, N'-DitridecylPeryleneDiimide based field-effect transistors: improving performance and stability with thermal treatment. Adv. Funct. Mater. 20, 2611. doi: 10.1002/adfm.201000383

Jeyaprakash, J. D., Samuel, S., and Ruhe, J. (2004). A facile photochemical surface modification technique for the generation of microstructured fluorinated surfaces. Langmuir 20, 10080-10085. doi: $10.1021 / \mathrm{la} 049428 \mathrm{~s}$

Komarneni, S. (1992). Feature article. Nanocomposites. J. Mater. Chem. 2, 1219-1230. doi: 10.1039/jm9920201219

Kuo, S. W., and Chang, F. C. (2011). POSS related polymer nanocomposites. Prog. Polym. Sci. 36, 1649-1696. doi: 10.1016/j.progpolymsci.2011.05.002

Laine, R. M. (2005). Nanobuilding blocks based on the [OSiO1.5] $\mathrm{x}$ $(\mathrm{x}=68,10)$ octasilsesquioxanes. J. Mater. Chem. 15, 3725-3744. doi: $10.1039 / \mathrm{b} 506815 \mathrm{k}$
Lee, Y. J., Kuo, S. W., Huang, C. F., and Chang, F. C. (2006). Synthesis and characterization of polybenzoxazine networks nanocomposites containing multifunctional polyhedral oligomeric silsesquioxane (POSS). Polymer 47, 4378-4386. doi: 10.1016/j.polymer.2006.03.111

Leu, C., Reddy, G. M., Wei, K., and Shu, C. (2003). Synthesis and dielectric properties of polyimide-chain-end tethered polyhedral oligomeric silsesquioxane nanocomposites. Chem. Mater. 15, 2261-2265. doi: $10.1021 / \mathrm{cm} 0208408$

Liang, J., He, L., Zhao, X., Dong, X., Luo, H., and Li, W. (2011). Novel linear fluoro-silicon-containing pentablock copolymers: synthesis and their properties as coating materials. J. Mater. Chem. 21, 6934-6943. doi: 10.1039/ cljm10635j

Liu, Y., Zheng, S., and Nie, K. (2005). Epoxy nanocomposites with octa(propylglycidyl ether) polyhedral oligomeric silsesquioxane. Polymer 46, 12016-12025. doi: 10.1016/j.polymer.2005.09.056

Nagendiran, S., Alagar, M., and Hamerton, I. (2010). 
Octasilsesquioxane-reinforced DGEBA and TGDDM epoxy nanocomposites: characterization of thermal, dielectric and morphological properties. Acta Materialia 58, 3345-3356. doi: 10.1016/j. actamat.2010.02.008

Phang, S. W., Tadokoro, M., Watanabe, J., and Kuramoto, N. (2009). Effect of $\mathrm{Fe} 3 \mathrm{O} 4$ and $\mathrm{TiO} 2$ addition on the microwave absorption property of polyaniline micro/nanocomposites. Polym. Advan. Technol. 20, 550-557. doi: $10.1002 /$ pat.1381

Qu, A., Wen, X., Pi, P., Cheng, J., and Yang, Z. (2008). Synthesis and characterization of hybrid fluoroemulsion based on silica/copolymer composite particles. Polym. Int. 57, 1287-1294. doi: 10.1002/pi.2477

Qu, L., and Xin, Z. (2011). Preparation and surface properties of novel low surface free energy fluorinated silane-functional polybenzoxazine films. Langmuir 27, 8365-8370. doi: 10.1021/la200073v

Raza, A., Si, Y., Wang, X., Ren, T., Ding, B., Yuc, J., et al. (2012). Novel fluorinated polybenzoxazine-silica films: chemical synthesis and superhydrophobicity. RSC Adv. 2, 12804-12811. doi: 10.1039/ c2ra21138f

Sanchez, C., Belleville, P., Popall, M., and Nicole, L. (2011a). Applications of advanced hybrid organicinorganic nanomaterials: from laboratory to market. Chem. Soc. Rev. 40, 696-753. doi: 10.1039/ c0cs00136h

Sanchez, C., Shea, K. J., and Kitagawa, S. (2011b). Recent progress in hybrid materials science. Chem. Soc. Rev. 40, 471-472. doi: 10.1039/ clcs $90001 \mathrm{c}$

Tang, Y., and Lewin, M. (2009). Migration and surface modification in polypropylene (PP)/polyhedral oligomeric silsequioxane (POSS) nanocomposites. Polym. Adv. Technol. 20, 1-15. doi: 10.1002/pat. 1229

Tsibouklis, J., and Nevell, T. G. (2003). Ultra-low surface energy polymers: the molecular design requirements. Adv. Mater. 15, 647-650. doi: 10.1002/adma.200301638

Vengatesan, M. R., Devaraju, S., Kannaiyan, D., Song, J. K., and Alagar, M. (2013). Ultrasoundassisted synthesis of benzoxazine monomers: thermal and mechanical properties of polybenzoxazines. Polym. Int. 62, 127-133. doi: 10.1002/pi.4337

Venkatesan, M. R., Devaraju, S., Ashok Kumar, A., and Alagar, M. (2011). Studies on thermal and dielectric properties of Octa (maleimido phenyl) silsesquioxane (OMPS) polybenzoxazine (PBZ) hybrid nanocomposites. High Perform. Polym. 23, 441-456. doi: 10.1177/ 0954008311414446

Venkatesan, M. R., Devaraju, S., Dinakaran, K., and Alagar, M. (2012). Studies on thermal and dielectric properties of organo clay and octakis (dimethylsiloxypropylglycidylether) silsesquioxane filled polybenzoxazine hybrid nanocomposites. Polym. Comp. 32, 1701-1711. doi: 10.1002/pc. 21177

Wang, L., Zhang, C., and Zheng, S. (2011). Organic-inorganic poly(hydroxyether of bisphenol A) copolymers with double-decker silsesquioxane in the main chains. J. Mater. Chem. 21, 19344-19352. doi: $10.1039 / \mathrm{cljm} 13596 \mathrm{a}$

Wu, S., Hayakawa, T., Kikuchi, R., Grunzinger, S. J., and Kakimoto, M. (2007). Synthesis and characterization of semiaromatic polyimides containing POSS in main chain derived from doubledecker-shaped silsesquioxane. Macromolecules 40, 5698-5705. doi: 10.1021/ma070547z

Xiang, K. M., and Chung, T. S. (2001). Effect of $-\mathrm{C}\left(\mathrm{CF}_{3}\right)_{2}$ - on the surface energy of main-chain liquid crystalline and crystalline polymers. J. Phys. Chem. B 105, 4145. doi: $10.1021 / \mathrm{jp} 003103 \mathrm{c}$

$\mathrm{Xu}, \mathrm{H} ., \quad K u o, \quad$ S., Lee, J., and Chang, F. (2002). Preparations, thermal properties, and $\mathrm{Tg}$ increase mechanism of inorganic/organic hybrid polymers based on polyhedral oligomeric silsesquioxanes. Macromolecules 35 , 8788-8793. doi: $10.1021 /$ ma0202843

Yoshida, N., Abe, Y., Shigeta, H., K.Takami, Osaki, H., and Watanabe, T. (2004). Preparation and water droplet sliding properties of transparent hydrophobic polymer coating by molecular design for self-organization. J. Sol Gel Sci. Technol. 31, 195-199. doi: 10.1023/ B:JSST.0000047986.92384.42

Zhang, W., and Müller, A. H. E. (2013). Architecture, self-assembly and properties of well-defined hybrid polymers based on polyhedral oligomeric silsequioxane (POSS). Prog. Polym. Sci. 38, 1121-1162. doi: 10.1016/ j.progpolymsci.2013.03.002

Zhang, Y. H., Lu, S. G., Li, Y. Q., Dang, Z. M., John, H. X., Fu, S. Y., et al. (2005). Novel silica tube/polyimide composite films with variable low dielectric constant. Adv. Mater. 17, 1056-1059. doi: 10.1002/adma.2004 01330

Zhuo, D., Gu, A., Liang, G., Hu, J., and Yuan, L. (2011). Preparation and properties of hollow silica tubes/cyanate ester hybrids for high-frequency copper-clad laminates. J. Mater. Sci. 46, 1571. doi: 10.1007/s10853-0104964-8

Zhuo, D., Gu, A., Wang, Y., Liang, G., Hu, J., Yuan, L., et al. (2012) Investigation of correlation between microstructure and dielectric properties of cyanate ester/silicate tube hybrids by positron annihilation lifetime spectroscopy. Polym Adv. Technol. 23, 1121-1128. doi: 10.1002/pat.2027

Conflict of Interest Statement: The authors thank BRNS, G. No: 2012/37C/9/BRNS, Mumbai, Govt. of India., for the financial support and Dr. Manmohan Kumar, Senior Scientific Officer, BARC, Mumbai for his support.

Received: 08 August 2013; paper pending published: 19 August 2013; accepted: 13 September 2013; published online: 14 October 2013.

Citation: Devaraju S, Prabunathan $P$, Selvi M and Alagar M (2013) Low dielectric and low surface free energy flexible linear aliphatic alkoxy core bridged bisphenol cyanate ester based POSS nanocomposites. Front. Chem. 1:19. doi: 10.3389/fchem.2013.00019

This article was submitted to Polymer Chemistry, a section of the journal Frontiers in Chemistry.

Copyright (c) 2013 Devaraju, Prabunathan, Selvi and Alagar. This is an open-access article distributed under the terms of the Creative Commons Attribution License (CC BY). The use distribution or reproduction in other forums is permitted, provided the original author(s) or licensor are credited and that the original publication in this journal is cited, in accordance with accepted academic practice. No use, distribution or reproduction is permitted which does not comply with these terms. 